UDC 517.5

D. V. Prokhorov

\title{
EXTENSION OF STARLIKE FUNCTIONS TO A FINITELY PUNCTURED PLANE
}

\author{
In memory of Alexander Vasil'ev
}

\begin{abstract}
We consider a sequence of functions which are starlike in the unit disk and their logarithmic derivatives are meromorphic with a finite number of simple poles in any boundary domain. These poles are either boundary deterministic or random with given characteristics. The aim of the article is the limit process and properties of the limit functions. We distinguish conditions for residues and distribution of poles. Under certain conditions, the sequence converges to the identity function. Another conditions allow us to obtain estimates for the limit function and its logarithmic derivative.
\end{abstract}

Key words: starlike function, meromorphic function, weight, deterministic point regulation, uniform distribution

2010 Mathematical Subject Classification: 30C45, 60G55

1. Introduction. During last decades stochastic ideas and methods became quite popular in geometric function theory. Among the most known events we mention that in 2000 Schramm introduced in [1] a family of random curves, which he called stochastic Loewner evolution, or SLE, see also [2] for further development.

In this article we present an approach to a (partly) stochastic problem posed by Vasil'ev for functions which are holomorphic in the plane, except for at most a countable set of singular points, and are starlike on the disk.

A function $f$ is said to be starlike if it maps the unit disk $\mathbb{D}=\{z \in$ $\in \mathbb{C}:|z|<1\}$ onto domain $f(\mathbb{D})$ which is starlike with respect to the origin. A holomorphic function $f$ on $\mathbb{D}$ normalized by $f(0)=0$ and $f^{\prime}(0)=1$ is starlike if and only if the real part of $z f^{\prime}(z) / f(z)$ is positive 
in $\mathbb{D}$ (see, e.g., [3, Theorem 2.10]). A dense subclass $\left\{f_{n}\right\}_{n \geq 1}$ of the set $S^{*}$ of all starlike functions $f$ is defined by

$$
\frac{z f_{n}^{\prime}(z)}{f_{n}(z)}=\sum_{k=1}^{n} \mu_{k n} \frac{a_{k}+z}{a_{k}-z}, \quad z \in \mathbb{D}
$$

where $\left|a_{k}\right|=1,0<\mu_{k n}<1, k=1, \ldots, n, \sum_{k=1}^{n} \mu_{k n}=1, n \in \mathbb{N}$.

In recent papers Alexander Vasil'ev developed stochastic topics in complex analysis, see, e.g., [4-6]. In early 2014 Vasil'ev proposed the problem of studying a limit process $f_{n} \rightarrow f$ as $n \rightarrow \infty$ when every starlike function $f_{n}$ is given by (1) with $\left|a_{k}\right| \geq 1, k=1, \ldots, n$, and $a_{n} \rightarrow \infty$ as $n \rightarrow \infty$.

Surely, we have to decide which conditions are required for a sequence of singular points $\left\{a_{n}\right\}_{n \geq 1}$. It seems interesting to consider the Poisson configuration of random points $\left\{a_{n}\right\}$, see, e.g., [7, $\left.\S 3.1\right]$.

Definition 1. The spatial Poisson process with uniform intensity $\beta>$ $>0$ is a point process in $\mathbb{R}^{2}$ that is a random distribution of points such that

[PP1] for every bounded closed set $B$, the count $N(B)$ which is the number of points of the process contained in $B$ has a Poisson distribution with the mean $\beta s(B)$, where $s(B)$ denotes the area of $B$;

[PP2] if $B_{1}, \ldots, B_{m}$ are disjoint regions, then $N\left(B_{1}\right), \ldots, N\left(B_{m}\right)$ are independent.

The Poisson process assumption relates only to the poles $\left\{a_{k}\right\}_{1 \leq k \leq n}$ in (1). However, there are families of coefficients $\left\{\mu_{k n}\right\}_{1 \leq k \leq n}, n \geq 1$, which are to be defined as well. We will prefer a deterministic way of choosing these weights. Let us describe two scenarios of weights behavior.

Given a sequence of numbers $\nu_{1}, \ldots, \nu_{n}, \ldots, 0<\nu_{n} \leq 1$ for all $n \geq 1$, denote

$$
S_{n}=\sum_{k=1}^{n} \nu_{k}, \quad \mu_{k n}=\frac{\nu_{k}}{S_{n}}, \quad 1 \leq k \leq n, \quad n \geq 1 .
$$

For every $n \geq 1$, we obtain a set of $n$ positive numbers $\mu_{1 n}, \ldots, \mu_{n n}$, $\sum_{k=1}^{n} \mu_{k n}=1$. There are only two possibilities:

(i) $S_{n} \rightarrow \infty$ as $n \rightarrow \infty$;

(ii) $\lim _{n \rightarrow \infty} S_{n}=S<\infty$.

In scenario (i), all weights $\mu_{k n}, 1 \leq k \leq n, n \geq 1$, tend to 0 uniformly as $n \rightarrow \infty$. This makes it sensible to call $\nu_{1}, \ldots, \nu_{n}, \ldots$ in (i) a uniform 
type sequence. For example $\left(\nu_{1}, \ldots, \nu_{n}, \ldots\right)=(1, \ldots, 1, \ldots)$ is a uniform type sequence. Indeed, in this case, $S_{n}=n$ for all $n \geq 1$, and

$$
\mu_{k n}=\frac{1}{n}, \quad 1 \leq k \leq n, \quad n \geq 1
$$

On the other hand, in scenario (ii) no one of weights $\mu_{k n}, n \geq 1$, tends to 0 for a fixed $k, 1 \leq k \leq n$, and $n \rightarrow \infty$, but $\mu_{k n}$ tend to 0 as $n \rightarrow \infty$ and $k \rightarrow \infty, 1 \leq k \leq n$. In this case, we call $\nu_{1}, \ldots, \nu_{n}, \ldots$ in (ii) a Poisson type sequence. For example,

$$
\left(\nu_{1}, \ldots, \nu_{n}, \ldots\right)=\left(1, \lambda, \ldots, \frac{\lambda^{n-1}}{(n-1) !}, \ldots\right), \quad \lambda>0,
$$

is a Poisson type sequence. Indeed, we see that $\lim _{n \rightarrow \infty} S_{n}=e^{\lambda}$ and therefore, for any fixed $k$,

$$
\lim _{n \rightarrow \infty}\left(\mu_{1 n}, \ldots, \mu_{k n}\right)=\left(e^{-\lambda}, e^{-\lambda} \lambda, \ldots, e^{-\lambda} \frac{\lambda^{k-1}}{(k-1) !}\right) .
$$

This is a sequence of values of a probability mass function of a discrete random variable having a Poisson distribution.

The article is organized as follows.

In Section 2 we deal with a deterministic point regulation, i.e., the points $\left\{a_{n}\right\}$ are determined and the weights $\left\{\mu_{k n}\right\}$ are generated by a sequence either of the uniform type or the Poisson type. We prove Theorem 1 , which states that $f_{n} \rightarrow$ id as $n \rightarrow \infty$ in the first case, and Theorem 2 with estimates for the limit functions in the second case.

In Section 3 we replace axioms $[\mathbf{P P 1}]-[\mathbf{P P 2}]$ by other ones which make the problem partly stochastic. Under the conditions of Theorem 3, functions $f_{n}$ converge almost surely to the identity.

2. Deterministic point regulation. Let $f_{n}, n \geq 1$, be given by (1) and denote

$$
p_{n}(z):=\frac{z f_{n}^{\prime}(z)}{f_{n}(z)}=1+\sum_{j=1}^{\infty} p_{j n} z^{j}, \quad z \in \mathbb{D} .
$$

We suppose that $\nu_{1}, \ldots, \nu_{n}, \ldots$ generating weights $\left\{\mu_{k n}\right\}$ is a uniform type sequence and show that $f_{n} \rightarrow$ id if $a_{n} \rightarrow \infty$ as $n \rightarrow \infty$.

Theorem 1. Given $\left\{a_{n}\right\}_{n \geq 1}$ with $\left|a_{n}\right| \geq 1$ and $a_{n} \rightarrow \infty$ as $n \rightarrow \infty$, let $f_{n}, n \geq 1$, be represented by (3) where the weights $\left\{\mu_{k n}\right\}_{1 \leq k \leq n<\infty}$ are 
generated according to (2) by a uniform type sequence $\nu_{1}, \ldots, \nu_{n}, \ldots$. Then $f_{n}$ converge to the identity function as $n \rightarrow \infty$ uniformly on compact subsets of $\mathbb{D}$.

Proof. Formulas (1) and (3) imply that coefficients $p_{j n}$ in (3) are evaluated as

$$
p_{j n}=2 \sum_{k=1}^{n} \frac{\mu_{k n}}{a_{k}^{j}}, \quad j \geq 1 .
$$

For any $\epsilon>0$, there exists $n_{1} \in \mathbb{N}$ such that

$$
\left|a_{n}\right|>1+\frac{1}{\epsilon}, \quad n>n_{1},
$$

and there exists $n_{2}>n_{1}$ for which

$$
0<\mu_{k n}<\frac{\epsilon}{n_{1}}, \quad n>n_{2} .
$$

Hence, for $n>n_{2}$ and any $j \geq 1$, we have

$$
\begin{gathered}
\left|p_{j n}\right| \leq 2 \sum_{k=1}^{n_{1}} \frac{\mu_{k n}}{\left|a_{k}^{j}\right|}+2 \sum_{k=n_{1}+1}^{n} \frac{\mu_{k n}}{\left|a_{k}^{j}\right|} \leq \\
\leq 2 \sum_{k=1}^{n_{1}} \mu_{k n}+2 \sum_{k=n_{1}+1}^{n} \mu_{k n} \frac{\epsilon}{1+\epsilon}<2 \epsilon+2 \epsilon=4 \epsilon .
\end{gathered}
$$

This means that, for any $j \geq 1, p_{j n}$ tend to 0 uniformly with respect to $j$ as $n \rightarrow \infty$, and thus $\lim _{n \rightarrow \infty} p_{n}(z)=1$. So $f_{n} \rightarrow$ id as $n \rightarrow \infty$ which completes the proof of Theorem 1 .

Remark 1. If $\left|a_{n}\right|=1+\beta n, \beta>0, n \geq 1$, then, for $f_{n}$ and $p_{n}$ in (3) under conditions of Theorem 1, the following relations

$$
\left|p_{n}(z)-1\right|=|z| O\left(\frac{1}{\sqrt{S_{n}}}\right), \quad\left|\log \frac{f_{n}(z)}{z}\right|=O\left(\frac{1}{\sqrt{S_{n}}}\right), \quad z \in \mathbb{D}, \quad n \rightarrow \infty,
$$

hold.

Indeed,

$$
\left|p_{n}(z)-1\right|=\left|\sum_{k=1}^{n} \frac{2 \mu_{k n} z}{a_{k}-1}\right| \leq \sum_{k=1}^{n} \frac{2 \mu_{k n}|z|}{\beta k}=\sum_{k=1}^{n} \frac{2 \nu_{k}|z|}{\beta k}\left[\sum_{k=1}^{n} \nu_{k}\right]^{-1} \leq
$$




$$
\begin{aligned}
& \leq 2|z| \sqrt{\sum_{k=1}^{n} \nu_{k}^{2}} \sqrt{\sum_{k=1}^{n} \frac{1}{(\beta k)^{2}}}\left[\sum_{k=1}^{n} \nu_{k}\right]^{-1} \leq \\
& \leq 2|z| \sqrt{\sum_{k=1}^{n} \frac{1}{(\beta k)^{2}}} \sqrt{\left[\sum_{k=1}^{n} \nu_{k}\right]^{-1}} \leq \frac{C|z|}{\sqrt{\sum_{k=1}^{n} \nu_{k}}}=\frac{C|z|}{\sqrt{S_{n}}}
\end{aligned}
$$

with a certain constant $C>0$.

This implies that

$$
\begin{aligned}
&\left|\log \frac{f_{n}(z)}{z}\right|=\left|\int_{0}^{z} \frac{p_{n}(\zeta)-1}{\zeta} d \zeta\right| \leq \int_{0}^{z} \frac{\left|p_{n}(\zeta)-1\right|}{|\zeta|}|d \zeta| \leq \\
& \leq \int_{0}^{|z|} \frac{C}{\sqrt{S_{n}}} d|\zeta|=\frac{C|z|}{\sqrt{S_{n}}}
\end{aligned}
$$

The situation is essentially different if $\nu_{1}, \ldots, \nu_{n}, \ldots$ generating weights $\left\{\mu_{k n}\right\}$ is a Poisson type sequence.

Theorem 2. Given $\left\{a_{n}\right\}_{n \geq 1}$ with $\left|a_{n}\right| \geq 1$ and $a_{n} \rightarrow \infty$ as $n \rightarrow \infty$, let $f_{n}, n \geq 1$, be represented by (3) where the weights $\left\{\mu_{k n}\right\}_{1 \leq k \leq n<\infty}$ are generated according to (2) by a Poisson type sequence $\nu_{1}, \ldots, \nu_{n}, \ldots$. Then $p=\lim _{n \rightarrow \infty} p_{n}$ and $f=\lim _{n \rightarrow \infty} f_{n}$ satisfy the following inequalities

$$
\begin{gathered}
\frac{2}{S} \sum_{k=1}^{\infty} \nu_{k} \frac{|z|}{\left|a_{k}\right|+|z|} \leq|p(z)-1| \leq \frac{2}{S} \sum_{k=1}^{\infty} \nu_{k} \frac{|z|}{\left|a_{k}\right|-|z|}, \quad z \in \mathbb{D} \\
|f(z)| \leq|z| \prod_{k=1}^{\infty}\left(1-\frac{|z|}{\left|a_{k}\right|}\right)^{-2 \nu_{k} / S}, \quad z \in \mathbb{D} .
\end{gathered}
$$

Proof. Formulas (1)-(3) imply that

$$
\begin{aligned}
p(z) & =\lim _{n \rightarrow \infty} p_{n}(z)=1+\lim _{n \rightarrow \infty} \sum_{j=1}^{\infty} p_{j n} z^{j}=1+\lim _{n \rightarrow \infty} \sum_{j=1}^{\infty} \sum_{k=1}^{n} \frac{2 \mu_{k n}}{a_{k}^{j}} z^{j}= \\
& =1+2 \lim _{n \rightarrow \infty} \sum_{k=1}^{n} \mu_{k n} \sum_{j=1}^{\infty}\left(\frac{z}{a_{k}}\right)^{j}=1+2 \lim _{n \rightarrow \infty} \sum_{k=1}^{n} \frac{\nu_{k}}{S_{n}} \frac{z}{a_{k}-z}=
\end{aligned}
$$




$$
=1+\frac{2}{S} \sum_{k=1}^{\infty} \nu_{k} \frac{z}{a_{k}-z} .
$$

From here we immediately deduce (4).

From the formula

$$
\log \frac{f(z)}{z}=\int_{0}^{z} \frac{p(\zeta)-1}{\zeta} d \zeta
$$

we find that

$$
\begin{gathered}
\log \left|\frac{f(z)}{z}\right| \leq\left|\log \frac{f(z)}{z}\right| \leq \int_{0}^{z} \frac{2}{S} \sum_{k=1}^{\infty} \nu_{k} \frac{|d \zeta|}{\left|a_{k}\right|-|\zeta|}= \\
=\frac{2}{S} \sum_{k=1}^{\infty} \nu_{k} \int_{0}^{|z|} \frac{d|\zeta|}{\left|a_{k}\right|-|\zeta|}=\frac{2}{S} \sum_{k=1}^{\infty} \nu_{k} \log \frac{\left|a_{k}\right|}{\left|a_{k}\right|-|z|}= \\
=\log \prod_{k=1}^{\infty}\left(1-\frac{|z|}{\left|a_{k}\right|}\right)^{-2 \nu_{k} / S}
\end{gathered}
$$

which implies estimate (5) and completes the proof of Theorem 2.

One can write down exact representations and estimates for the sequence $\nu_{1}, \ldots, \nu_{n}, \ldots$ with the Poisson distribution.

3. Random point regulation. In this section, we deal with a random model of point configurations based on the Poisson process of Definition 1. However, we will transform this model in accordance with the specific problem for starlike functions having random singular points in the plane.

First, we have seen in the previous sections that estimates of Theorems 1 and 2 are independent of $\arg a_{n}$. Therefore, it is natural to apply the one-dimensional Poisson process on the line, i.e., we assume that $a_{n} \geq 1$ for $n \geq 1$.

Secondly, we focus on a homogeneous Poisson point process which is also called a stationary Poisson process, see, e.g., [8, pp. 19-25], as well as uniform Poisson point process, see, e.g., [9, p.13]. If the homogeneous point process is defined on the real line in different mathematical models, then it has the characteristic that the positions will be uniformly distributed. 
Third, a compound Poisson point process is formed by adding random values or weights to each point of the Poisson point process, so the process is constructed from a marked Poisson point process, where the marks form a collection of independent and identically distributed non-negative random values, see, e.g., [10, pp. 19-21; 11, pp. 291-293]. In our case, the weights $\left\{\mu_{k n}\right\}$ serve as deterministic marks either of the uniform type or the Poisson type.

Finally, we replace the axiom [PP1] in Definition 1 by a deterministic number of points in a given interval.

So we make the following assumptions.

[UP1] For every $R>1$, there is a number $n(R)$ of points $\left\{a_{k}\right\}_{1 \leq k \leq n(R)}$ which arrive in the segment $[1, R]$.

[UP2] Random points $\left\{a_{k}\right\}_{1 \leq k \leq n(R)}$ are uniformly distributed in $[1, R]$, knowing the number $n(R)$.

[UP3] Random points $\left\{a_{k}\right\}$ are independent.

Theorem 3. Suppose that a random point process satisfies axioms [UP1] - [UP3], and let functions $f_{n(R)}$ be represented by (1) and (3) with arbitrary positive numbers $\left\{\mu_{k n(R)}\right\}_{1 \leq k \leq n(R)}, \sum_{k=1}^{n(R)} \mu_{k n(R)}=1$. Then $f_{n(R)}$ converge almost surely to the identity function $f(z)=z$ as $R \rightarrow \infty$.

Proof. Formulas (1) and (3) imply that

$$
p_{n(R)}(z)=1+\sum_{j=1}^{\infty} p_{j n(R)} z^{j}, \quad z \in \mathbb{D},
$$

where

$$
p_{j n(R)}=\sum_{k=1}^{n(R)} \frac{2 \mu_{k n(R)}}{a_{k}^{j}} .
$$

As far as random points $\left\{a_{k}\right\}_{1 \leq k \leq n(R)}$ are independent, a joint probability density function $\varphi\left(a_{1}, \ldots, a_{n(R)}\right)$ is evaluated as follows

$$
\varphi\left(a_{1}, \ldots, a_{n(R)}\right)=\prod_{k=1}^{n(R)} \varphi\left(a_{k}\right),
$$

where $\varphi\left(a_{k}\right)$ is a probability density function for the random point $a_{k}$. Since $a_{k}$ is uniformly distributed in $[1, R]$, its probability density function 
has the form

$$
\varphi\left(a_{k}\right)=\frac{1}{R-1} \text { for } a_{k} \in[1, R], \text { and } \varphi\left(a_{k}\right)=0 \text { for } a_{k} \notin[1, R],
$$

$k=1, \ldots, n(R)$.

Evaluate the mathematical expectation $\mathbb{E}\left(p_{j n(R)}\right)$ of $p_{j n(R)}, j \geq 1$,

$$
\begin{aligned}
& \mathbb{E}\left(p_{j n(R)}\right)=\int_{1}^{R} \ldots \int_{1}^{R} \sum_{k=1}^{n(R)} \frac{2 \mu_{k n(R)}}{a_{k}^{j}} \varphi\left(a_{1}, \ldots, a_{n(R)}\right) d a_{1} \ldots d a_{n(R)}= \\
& =\sum_{k=1}^{n(R)} 2 \mu_{k n(R)} \int_{1}^{R} \frac{\varphi\left(a_{k}\right)}{a_{k}^{j}} d a_{k}=\sum_{k=1}^{n(R)} \frac{2 \mu_{k n(R)}}{R-1} \int_{1}^{R} \frac{d a_{k}}{a_{k}^{j}}=\frac{2}{R-1} \int_{1}^{R} \frac{d x}{x^{j}} .
\end{aligned}
$$

Hence,

$$
\mathbb{E}\left(p_{1 n(R)}\right)=\frac{2 \log R}{R-1}
$$

and

$$
\mathbb{E}\left(p_{j n(R)}\right)=\frac{2}{R-1} \frac{R^{j-1}-1}{(j-1) R^{j-1}}, \quad j>1 .
$$

So, for all $j \geq 1$,

$$
\lim _{R \rightarrow \infty} \mathbb{E}\left(p_{j n(R)}\right)=0,
$$

and the convergence is uniform with respect to $j \geq 1$. This implies that $p_{n(R)}$ converge almost surely to $p(z) \equiv 1$ as $R \rightarrow \infty$, and hence $f_{n(R)}$ converge almost surely to $f(z)=z, z \in \mathbb{D}$, as $R \rightarrow \infty$, which completes the proof of Theorem 3 .

Note that axiom [UP1], as well as Theorem 3 and its proof, do not require that $n(R) \rightarrow \infty$ when $R \rightarrow \infty$.

Evidently, requirement [UP2] about the uniform distribution of points $\left\{a_{k}\right\}$ in $[1, R]$ can be replaced by other suitable continuous distributions, e.g., normal, exponential, geometric stable and so on. Discrete distributions are possible as well. Certainly, the conclusion of Theorem 3 can be changed under different assumptions.

For example, let $\varphi\left(a_{k}\right)$ be a probability density function with parameter $\lambda>0$ for the exponential distribution located on $[1, R], R>1$,

$$
\varphi(x)=\frac{\lambda e^{-\lambda(x-1)}}{1-e^{-\lambda(R-1)}}, \quad x \in[1, R]
$$


and $\varphi(x)=0$ outside $[1, R]$.

Following the proof of Theorem 3 in this case, we come to the formula

$$
\mathbb{E}\left(p_{j n(R)}\right)=2 \int_{1}^{R} \frac{\varphi(x)}{x^{j}} d x=\frac{2 \lambda}{1-e^{-\lambda(R-1)}} \int_{1}^{R} \frac{e^{-\lambda(x-1)}}{x^{j}} d x, \quad j \geq 1 .
$$

Send $R$ to infinity and obtain

$$
\lim _{R \rightarrow \infty} \mathbb{E}\left(p_{j n(R)}\right)=\frac{2}{\lambda} \int_{1}^{\infty} \frac{e^{-\lambda(x-1)}}{x^{j}} d x>0, \quad j \geq 1 .
$$

In such a case, if $\left\{a_{k}\right\}_{1 \leq k \leq n(R)}$ has a limit configuration $\left\{a_{n}\right\}_{n=1}^{\infty}$ as $R \rightarrow \infty$, then the almost surely limit $f$ is a starlike function in $\mathbb{D}$, and $p(z)=z f^{\prime}(z) / f(z)$ is meromorphic with simple poles at $\left\{a_{n}\right\}_{n=1}^{\infty}$.

Acknowledgment. This paper is dedicated to the memory of Alexander Vasil'ev (1962-2016), author's first and the best PhD student. The article was partially prepared during author's stay at Bergen University in Norway during January-April, 2014, under invitation by Vasil'ev who proposed the research topic and actively participated in the work. A part of results was announced in [12] at the international conference "Function Theory, its Applications and Related Topics", Kazan Federal University, 2015.

This work is supported by the Russian Science Foundation under grant 17-11-01229.

\section{References}

[1] Schramm O. Scaling limits of loop-erased random walks and uniform spanning trees. Israel J. Math., 2000, vol. 118, pp. 221-288. DOI: 10.1007/BF02803524.

[2] Lawler G. F. Conformally Invariant Processes in the Plane. Mathematical Surveys and Monographs, vol. 114, American Mathematical Society, 2005.

[3] Duren P. L. Univalent Functions. Springer-Verlag, 1983.

[4] Ivanov G., Kang N.-G., Vasil'ev A. Slit holomorphic stochastic flows and Gaussian free field. Complex Anal. Oper. Theory, 2016, vol. 10, no. 7, pp. 1591-1617. DOI: 10.1007/s11785-016-0536-5. 
[5] Bracci F., Contreras M. D., Díaz-Madrigal S., Vasil'ev A. Classical and stochastic Löwner-Kufarev equations. Vasil'ev A. (ed.): Harmonic and Complex Analysis and its Applications. Trends Math., Birkhäuser, 2013, pp. 39-134. DOI: 10.1007/978-3-319-01806-5_2.

[6] Ivanov G., Vasil'ev A. Löwner evolution driven by a stochastic boundary point. Anal. Math. Phys., 2011, vol. 1, no. 4, pp. 387-412. DOI: 10.1007/s13324-011-0019-9.

[7] Baddeley A. Spatial point patterns: models and statistics. Spodarev E. (ed.): Stochastic geometry, spatial statistics and random fields. Lecture Notes Math., vol. 2068, Springer, 2013, pp. 49-114. DOI: 10.1007/978-3642-33305-7_3.

[8] Daley D. J., Vere-Jones D. An Introduction to the Theory of Point Processes. Vol. II: General Theory and Structure. Springer Science and Business Media, 2007.

[9] Kingman J. F. C. Poisson Processes. Clarendon Press, 1992.

[10] Baddeley A., Bárány I., Schneider R. Stochastic Geometry: Lectures given at the C.I.M.E. Summer School held in Martina Franca, Italy, Sept., 13-18, 2004. Springer, 2006.

[11] Baccelli F., Blaszczyszyn B. Stochastic Geometry and Wireless Networks. Now Publisher Inc., 2009.

[12] Prokhorov D. Functions with a Poisson configuration of singular points. Function Theory, its Applications and Related Topics. Abstracts XII Kazan International Summer Scientific Conference (Kazan, June, 27 - July, 4), 2015, pp. 357-359.

Received May 23, $201 \%$.

In revised form, June 7, $201 \%$.

Accepted June 10, $201 \%$.

Published online June 10, $201 \%$.

Saratov State University,

83, Astrakhanskaya st., Saratov 410012, Russia;

Petrozavodsk State University

33, Lenina pr., Petrozavodsk 185910, Russia

E-mail: prokhorovdv@info.sgu.ru 